\title{
Ligação de um instrumento de avaliação clínica de exposição ao vírus Zika com a Classificação Internacional de Funcionalidade, Incapacidade e Saúde
}

\section{Linking a clinical assessment instrument for Zika virus exposure with the International Classification of Functioning, Disability and Health}

(D) Nathália Cristina Oliveira de Souza ${ }^{1}$, (DLara Carolina Januário Cabral ${ }^{1}$, (DLaura Cristina Machado Ribeiro de Souza ${ }^{1}$, (DMarcos Vinicius da Silva Pone ${ }^{1}$, (DLuciana Castaneda Ribeiro ${ }^{2}$, (D) Carla Trevisan Martins Ribeiro ${ }^{1}$

\begin{abstract}
RESUMO
Instrumentos de avaliação clínica foram criados a partir de experiências prévias de cuidados para outras condições crônicas complexas da infância, de modo a planejar e sistematizar ações. Contudo, é importante que tais instrumentos tenham validação externa e contemplem o atual conceito de saúde biopsicossocial. Uma interlocução com a Classificação Internacional de Funcionalidade, Incapacidade e Saúde (CIF) poderia dar subsídio teórico e validação aos instrumentos criados. Objetivo: Identificar o conteúdo comum da CIF com o instrumento de avaliação clínica aplicado em população exposta ao vírus Zika em um ambulatório de doenças infecciosas em pediatria de um hospital de referência no Estado do Rio de Janeiro. Métodos: A Ligação com a CIF foi realizada por dois revisores independentes, segundo a proposta de Cieza e colaboradores. O coeficiente Kappa foi utilizado para análise da concordância interobservadores. Resultados: 0 instrumento de avaliação clínica utilizado no ambulatório de referência é composto principalmente por categorias da CIF de estruturas do corpo (46,4\%). Observou-se poucas categorias relacionadas aos fatores contextuais $(13,1 \%)$. E, não foram encontrados itens relacionados às categorias de atividade e participação. Conclusão: A ferramenta de avaliação apresenta principalmente informações sobre as funções e estruturas do corpo, voltando-se a um olhar puramente restrito as funções fisiológicas e as estruturas anatômicas. A inexistência de categorias de atividade e participação pode comprometer a percepção das experiencias vividas pelas crianças que foram expostas ao vírus Zika.
\end{abstract}

Palavras-chave: Cuidado da Criança, Avaliação em Saúde, Zika Virus, Classificação Internacional de Funcionalidade, Incapacidade e Saúde

\section{ABSTRACT}

The Zika epidemic and the emergence of a new health condition in Brazil, imposed a rapid organization on services to meet the current demand for care. Clinical assessment instruments were created, based on previous care experiences for other complex chronic conditions in order to plan and systematize actions. However, it is important that these instruments have external validation and include the current concept of health, biopsychosocial. An interlocution with the International Classification of Functioning, Disability and Health (CIF) could provide theoretical support and validation to the instruments created. Objective: To identify the common content among the clinical evaluation instrument applied to the population exposed to the Zika virus in a pediatric infectious disease outpatient clinic in a reference hospital in the State of Rio de Janeiro with the ICF. Methods: The link with the ICF was carried out by two independent reviewers, according to Cieza's proposal and the Kappa coefficient was used for interobserver analysis. Results: It was identified that the instrument is mainly composed of items of body structure $(39 ; 46.4 \%)$. There were few categories related to contextual factors $(11 ; 13.1 \%)$. And there were no items related to the categories of activity and participation. Conclusion: The assessment tool presents mainly domains of function and structure of the body, turning to a biomedical look. The lack of categories of activity and participation can compromise the perception of health status. A line of care in the light of the biopsychosocial model provides advantages for health services planning and actions

Keywords: Child Care, Health Evaluation, Zika Virus, International Classification of Functioning, Disability and Health 


\section{INTRODUÇÃO}

Após a epidemia de Zika vírus no Brasil e sua associação com o aumento do número de casos de microcefalia congênita no final de 2015, os serviços de saúde brasileiros tiveram que se estruturar rapidamente para dar conta desta nova demanda. ${ }^{1,2}$

Crianças expostas ao vírus Zika durante a gestação podem apresentar importantes alterações no sistema nervoso central (SNC), comorbidades ortopédicas e oftalmológicas ocasionando a Síndrome Congênita pelo Zika vírus (SCZ); ou apenas características mais sutis na linguagem ou na motricidade percebidas somente ao longo do desenvolvimento., ${ }^{3,4}$ Estas manifestações de maior ou menor grau repercutem sobre o crescimento e desenvolvimento e demandam avaliação criteriosa e cuidados durante toda a vida. ${ }^{5,6}$

Assim, com o súbito aparecimento desta nova infecção congênita, os serviços de saúde precisaram se organizar para atender as demandas de cuidado da criança e de sua família, criando instrumentos específicos de avaliação e acompanhamento clínico. Contudo, atualmente os instrumentos de avaliação em saúde devem contemplar informações orientadas ao modelo biopsicossocial preconizado no atual cuidado de saúde. Estes instrumentos também precisam de validação externa, com padronizações de

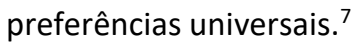

Uma interlocução com a Classificação Internacional de Funcionalidade, Incapacidade e Saúde (CIF) poderia dar subsídio teórico e unificar a linguagem através da codificação dos procedimentos acerca da funcionalidade. A CIF tem objetivo de possibilitar uma linguagem universal para descrição de problemas de saúde e suas intervenções ${ }^{8}$ e pode ser utilizada como modelo de orientação do entendimento das medidas de resultado a partir das respostas de pacientes. ${ }^{9}$

A funcionalidade, a partir desta perspectiva corresponde aos aspectos positivos da condição de saúde que envolve as funções e estruturas do corpo, atividade e participação, numa interação direta com os fatores contextuais (ambientais e pessoais) (Figura1). ${ }^{8}$

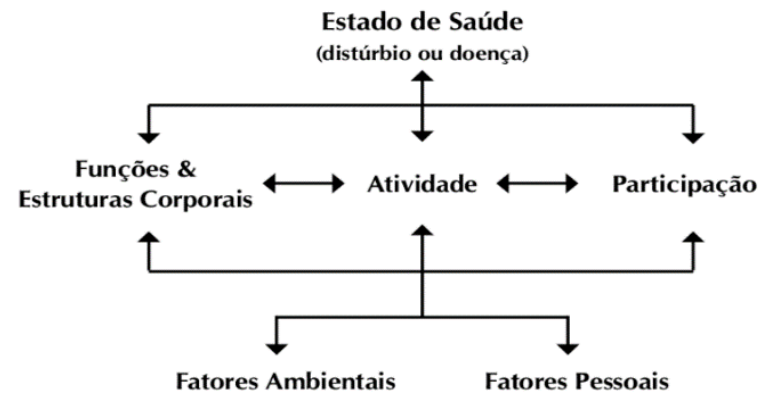

Fatores Contextuais

Figura 1. Modelo de interação dos conceitos da CIF. ${ }^{8}$

Utilizar instrumentos clínicos de avaliação com base na CIF que tenham a capacidade de cobertura de informações centradas no usuário e na sua família podem garantir melhor abrangência no cuidado a saúde. ${ }^{10}$ Estudos de ligação de instrumentos de avaliação com a CIF são amplamente difundidos na literatura. As evidências geradas por esse tipo de metodologia potencializam exemplos de utilização da CIF na prática clínica. ${ }^{9,11}$ A uniformidade da linguagem clínica com foco na Funcionalidade possibilita qualificação da assistência ao paciente, além da melhoria da comunicação entre os profissionais envolvidos com a reabilitação e o cuidado ambulatorial.

\section{OBJETIVO}

O objetivo deste estudo é realizar a identificação de conteúdo comum entre a CIF e o instrumento de avaliação clínica aplicada na população exposta ao vírus Zika de um ambulatório de doenças infecciosas em pediatria em um ambulatório de referência da alta complexidade no Estado do Rio de Janeiro.

\section{MÉTODOS}

Estudo metodológico realizado a partir dos instrumentos de avaliação clínica de um ambulatório multiprofissional para assistência às crianças expostas ao Zika vírus durante a gestação, de um hospital materno-infantil de referência do estado do Rio de Janeiro. A identificação de conteúdo comum dos instrumentos de avaliação clínica ocorreu a partir de três etapas distintas: 1) identificação de conteúdo significativo e posteriormente comum dos desfechos do instrumento com a CIF, 2) comparação entre os resultados da identificação, e 3) análise estatística dos dados.

A identificação do conteúdo comum entre o instrumento de avaliação clínica e a CIF foi realizada por dois revisores independentes de forma cega. A comparação das ligações dos dois pesquisadores foi efetuada ao final de cada etapa e para as categorias divergentes, um terceiro revisor foi consultado para consenso, conforme as regras de ligação propostas por Cieza. ${ }^{12}$ Todos os revisores eram previamente familiarizados com a CIF.

Inicialmente, foi realizada a análise do instrumento clínico utilizado pelos médicos (instrumento de primeira avaliação e de acompanhamento), e os itens pertencentes aos instrumentos que estavam relacionados a avaliação da criança foram identificados, sendo eles desfechos de identificação, classificação da exposição, história familiar, história perinatal, exames laboratoriais, exames complementares, uso de tecnologia assistiva, história medicamentosa, vínculos a outros serviços, exame físico, avaliação genética, cardiológica, oftalmológica, neurológica, nutricional e de desenvolvimento.

Após a extração dos conteúdos dos instrumentos de avaliação clinica utilizados pelos médicos no ambulatório foram extraídos os conceitos significativos de cada desfecho que foram ordenados em uma planilha de $\mathrm{Excel}^{\circledR}$.

A versão do livro da CIF do ano de 2015 foi utilizada como base. Esta versão está disponível em português e agrupa a classificação pediátrica com a do adulto. Além disso, seguiu-se a proposta de Cieza et al. ${ }^{12}$ que descreveram regras que garantem clareza e confiabilidade durante a associação. ${ }^{8} \mathrm{O}$ conjunto de regras foram atualizadas no ano de 2016 e estão dispostas no Quadro 1.

O sistema hierárquico da CIF é organizado por capítulos, seguidos de categorias de segundo, terceiro e quarto nível. Com a progressão dos níveis, existe maior detalhamento das 
informações. ${ }^{12} \mathrm{O}$ presente estudo optou por realizar as ligações em segundo nível da CIF e somente com os conceitos principais. Esse nível será utilizado por especificar as características da incapacidade e ainda descrever suas definições. ${ }^{8}$

Para análise estatística dos dados foi utilizado o coeficiente Kappa, que verifica a concordância da ligação entre avaliadores. $O$ índice gerado, corresponde a capacidade de dois ou mais aplicadores alcançarem os mesmos resultados, estabelecendo confiabilidade interobservadores e indicando a qualidade da ligação. ${ }^{13} \mathrm{~A}$ interpretação é dada como, zero, equivalência pobre; $>0$ a 0,19, leve; de 0,20 a 0,39, considerável; 0,40 a 0,59, moderada; 0,60 a 0,79, substancial e 0,80 a 1 , excelente. ${ }^{14}$

\section{Quadro 1. Regras de vinculação da CIF $^{12}$}

\begin{tabular}{|c|c|}
\hline 1 & $\begin{array}{l}\text { Conhecimento sobre os conceitos, capítulos, domínios e } \\
\text { categorias da CIF, para em seguida identificar os } \\
\text { conceitos significativos do estado de saúde. }\end{array}$ \\
\hline 2 & $\begin{array}{l}\text { Identificar sobre o que se trata o item. Ajudando a } \\
\text { identificar o conceito significativo mais relevante. }\end{array}$ \\
\hline 3 & $\begin{array}{l}\text { Identificar conceitos adicionais além dos conceitos } \\
\text { principais da etapa anterior. }\end{array}$ \\
\hline 4 & $\begin{array}{l}\text { Identificar e documentar as perspectivas relacionadas à } \\
\text { saúde. }\end{array}$ \\
\hline 5 & $\begin{array}{l}\text { Identificar e documentar as opções de resposta caso o } \\
\text { instrumento as tenha. }\end{array}$ \\
\hline 6 & $\begin{array}{l}\text { Vincular todos os conceitos significativos (principais e } \\
\text { adicionais) a categoria da CIF que melhor o abrange. }\end{array}$ \\
\hline 7 & $\begin{array}{l}\text { Utilizar a categoria "outro especificado" ou "não } \\
\text { especificado" quando necessário. }\end{array}$ \\
\hline 8 & $\begin{array}{l}\text { Se a informação do conceito significativo não for } \\
\text { suficiente para classificar em uma categoria, atribuir o } \\
\text { conceito (nd- não definível). }\end{array}$ \\
\hline 9 & $\begin{array}{l}\text { Se o conceito significativo não for coberto pela CIF mas } \\
\text { for um fator pessoal, atribuir o conceito (fp- fator } \\
\text { pessoal). }\end{array}$ \\
\hline 10 & $\begin{array}{l}\text { Se o conceito significativo não for coberto pela CIF, } \\
\text { atribuir o conceito (nc-não coberto). } \\
\text { Se o conceito significativo se referir a um diagnóstico ou } \\
\text { condição de saúde, atribuir o conceito (nc-hc - não } \\
\text { coberto- condição de saúde). } \\
\text { Se o conceito significativo se referir a qualidade de vida, } \\
\text { atribuir o conceito (nc-qol - não coberto-qualidade de } \\
\text { vida). }\end{array}$ \\
\hline
\end{tabular}

\section{RESULTADOS}

O instrumento de avaliação clínica do ambulatório de pediatria é composto por oitenta e quatro desfechos. A identificação de conteúdo comum com a CIF identificou que a maior parte dos itens $(39 ; 46,4 \%)$ foram relacionados a domínios de estrutura do corpo.
Observou-se também domínios correspondentes a fatores pessoais $(11 ; 13,1 \%)$, contudo não foram observados desfechos que contivessem informações sobre categorias de atividade e participação, como pode ser observado na Tabela 1. A análise do coeficiente Kappa para avaliação interobservador apresentou valor 0,62 que corresponde a uma ligação substancial ic $(0,60-0,65)$.

Tabela 1. Descrição dos componentes presentes no instrumento de avaliação médica utilizado em um ambulatório de atendimento a crianças com ZKV e os componentes da CIF, Rio de Janeiro, 2019

\begin{tabular}{lcc}
\hline Componente da CIF & $\mathbf{n}$ & \% \\
\hline Função do Corpo & 20 & 23,8 \\
Estrutura do Corpo & 39 & 46,4 \\
Fator Ambiental & 13 & 15,5 \\
Fator Pessoal & 11 & 13,1 \\
Condição de Saúde & 1 & 1,2 \\
Total & 84 & 100 \\
\hline
\end{tabular}

Nota: n: número de itens; \%:porcentagem

Alguns desfechos do instrumento no processo de identificação de conteúdo comum com a CIF se repetiram. Os mais frequentes relacionados a função do corpo foram b230 (funções auditivas) e b540 (funções metabólicas gerais). As funções auditivas estavam relacionadas aos itens da ficha clínicas referentes a exames auditivo (como PEAT - Potencial Evocado Auditivo de Tronco Cefálico e EOAT - Emissões Auto Acústicas Transientes) já os da função metabólica com os vários exames de sangue específicos.

Outras categorias importantes para a criança exposta a Zika como função urinária (b620), tônus muscular (b730) e fluência e ritmo da fala (b330), estavam relacionadas ao acompanhamento e exames de urodinâmica, ao exame físico e neurológico, e avaliação de linguagem, respectivamente. Já a informação do exame de eletroencefalograma foi ligada a categoria de função da consciência (b110).

Para as categorias de estruturas do corpo, os códigos $\mathbf{s 1 1 0}$ e s710 foram os mais encontrados e referem-se a estrutura do cérebro e estrutura da cabeça e pescoço. No instrumento de avaliação clínica estes códigos se relacionam a exames de imagem (como ultrassonografia transfontanela, tomografia computadorizada etc.) e exame físico de cabeça e pescoço, além da medição de perímetro cefálico.

Já em fatores ambientais as categorias e110 (produtos e substâncias de consumo pessoal) foram relacionados a questões referente a acesso a medicação e alimentação e o e580 (serviços, sistemas e políticas de saúde) que inclui coleta de informações acerca do acesso a tratamentos específicos (fisioterapia, fonoaudiologia, terapia ocupacional etc) e consultas médicas especializadas (neurologia). Todos os domínios encontrados no instrumento de avaliação estão no Quadro 2.

Os fatores pessoais que até o momento não possuem códigos de cobertura pela CIF foram considerados nos conteúdos do instrumento de avaliação clínica nos desfechos de história do desenvolvimento motor, história gestacional, raça e gênero. 
Quadro 2. Domínios da CIF encontrados no instrumento de avaliação clínica

\begin{tabular}{|c|c|c|c|}
\hline \multicolumn{2}{|r|}{ ESTRUTURA } & \multicolumn{2}{|r|}{ FUNÇÃO } \\
\hline Código & Significado & Código & Significado \\
\hline s110 & Estrutura do cérebro & $b 110$ & Funções da consciência \\
\hline$s 210$ & estrutura da cavidade orbital & b230 & Funções auditivas \\
\hline$s 220$ & Estrutura do bulbo do olho & b330 & Funções da fluência e ritmo da fala \\
\hline s320 & Estrutura da boca & $b 440$ & Funções respiratórias \\
\hline$s 420$ & Estrutura do sistema imunológico & b530 & Funções de manutenção do peso \\
\hline$s 430$ & Estrutura do sistema respiratório & $b 540$ & Funções metabólicas gerais \\
\hline s5 & Estrutura do sistema digestório & $b 620$ & Funções urinárias \\
\hline$s 540$ & Estrutura do intestino & $b 710$ & Função de mobilidade das articulações \\
\hline$s 560$ & Estrutura do fígado & b735 & Funções de tônus muscular \\
\hline$s 630$ & Estrutura do sistema genital & b750 & Funções de reflexo motor \\
\hline s7 & Estrutura relacionada ao movimento & & Fatores Ambientais \\
\hline$s 710$ & Estrutura da região da cabeça e pescoço & e110 & Produtos e substâncias de consumo pessoal \\
\hline$s 730$ & Estrutura da extremidade superior & e115 & Produtos e tecnologia para uso pessoal na vida diária \\
\hline$s 740$ & Estrutura da região pélvica & e125 & Produtos e tecnologia para comunicação \\
\hline$s 750$ & Estrutura da extremidade inferior & e165 & Bens \\
\hline s760 & Estrutura do tronco & e575 & Serviços, sistemas e políticas de suporte social \\
\hline s810 & Estrutura da pele & $e 580$ & Serviços, sistemas e políticas de saúde \\
\hline
\end{tabular}

\section{DISCUSSÃO}

A partir da ligação do instrumento de avaliação clínica para crianças expostas ao vírus Zika com a CIF foi possível identificar que a documentação das informações é composta principalmente por categorias de função do corpo e estrutura do corpo (70,2\%). Não foram encontradas categorias de atividade e participação. Isto reflete que o instrumento de avaliação, apesar de extenso ainda está ancorado em um olhar biomédico.

O modelo biomédico leva em consideração as deficiências e doenças e como esses fatores geram a incapacidade, ou seja, o olhar é voltado para o corpo deficiente. E, o impacto gerado a partir desta categorização leva ao profissional uma necessidade de reparar um desvio da normalidade, criando dessa forma uma barreira social quando esse reparo não é possível. ${ }^{15}$ É importante compreender a complexidade do indivíduo e a necessidade de garantir a avaliação de todos os aspectos de sua vida. ${ }^{16}$

Os resultados identificaram que as categorias mais prevalentes de estrutura e função foram as relacionados a estrutura da cabeça e encéfalo e função auditiva e metabólicas.

Isto é compatível com o conhecimento já adquirido sobre a Zika que demonstra além da microcefalia, outras malformações cerebrais como calcificações subcorticais, ventriculomegalia, epilepsia, entre outros, além de alterações auditivas em crianças expostas ao vírus zika com ou sem SCZ. 4,6,17

A inexistência de categorias que avaliem a atividade e participação, demonstra ao registro parcial da avaliação clínica e compromete a percepção da condição de saúde em sua totalidade. É importante compreender que o conceito biopsicossocial trazido pela CIF conceitualiza a condição de saúde como uma relação dinâmica entre as estruturas e as funções do corpo, com as atividades desempenhadas e participação do indivíduo no meio social. ${ }^{8}$
Nas crianças expostas ao vírus Zika as manifestações clínicas observadas a partir de prejuízo claros de estrutura anatômicas e função corporais levam a limitação de atividades como aquisição de linguagem e mobilidade e gera restrições na participação social do indivíduo e sua família, como ir à escola ou brincar. ${ }^{18-20}$

0 estudo de Ferreira, $^{20}$ relata que 0 atraso no desenvolvimento neuropsicomotor apresentado pelas crianças expostas ao vírus Zika repercute negativamente nas atividades de vida diária e lazer, interferindo a longo prazo na saúde e qualidade de vida. No mesmo estudo, a mobilidade foi identificada como um dos principais desafios pela família e equipe de saúde, levando a uma perda de interação entre a criança e seu ambiente. Portanto, uma avaliação e acompanhamento precoce que identifique a necessidade do indivíduo, impacta diretamente na sua funcionalidade.

Com relação aos fatores contextuais, poucos aspectos foram encontrados na presente pesquisa. As categorias observadas concentraram-se no acesso a produtos de consumo pessoal (como leite, medicação, acesso à tecnologia assistiva etc.) e a serviço de saúde (como acesso a reabilitação, especialidades médicas e exames).

Com relação a isto, uma revisão sistemática de 2018, traz os fatores contextuais como uma categoria que impacta diretamente a qualidade de vida de famílias atingidas pela SCZV. ${ }^{18}$ Um outro estudo de 2019, relata que os aspectos contextuais, principalmente relacionados ao acesso serviço de saúde, a relação interprofissional e as condições de vida da família impactam sobre o cuidado da criança e sua condição de saúde e devem ser considerados. ${ }^{3}$ Por isso a importância da avaliação do componente de fatores contextuais (especialmente os ambientais) na avaliação de saúde dos indivíduos. Para crianças expostas ao vírus Zika, que apresentam uma variedade de acometimentos, utilizar 
instrumentos de avaliação que analisem a condição clínica e os aspectos contextuais potencializa o processo de cuidado, pois já se conhece a influência dos fatores ambientais no desenvolvimento neuropsicomotor dessa população. ${ }^{21}$

Vale destacar que a construção do instrumento de avaliação clínica analisado se deu no início da pandemia pelo Zika vírus, e com o conhecimento que se tinha sobre a condição de saúde recém descrita. Sua finalidade era o registro sobre a apresentação clínica da doença. Além disso, uma vez que a avaliação clínica foi criada pela equipe médica, era esperado o predomínio do conceito biomédico. Contudo, a assistência à criança com zika neste ambulatório é multiprofissional, e estes possuíam seus próprios instrumentos de avaliação. Desta forma, é preciso ponderar que os itens não contemplados na ficha clínica podem estar presente nas fichas das demais especialidades. Contudo uma vez que o hospital analisado também é um hospital de pesquisa e ensino e há uma linha de pesquisa em desenvolvimento específica sobre o tema, seria possível uma avaliação mais abrangente.

Assim, com o conhecimento atual sobre a importância da avaliação global e a contribuição advinda do aporte teórico da CIF, faz-se necessário a implementação de domínios relacionados a funcionalidade no instrumento de avaliação clínica. Complementar a dualidade do modelo biomédico com um olhar mais amplo, que observa os impactos da doença nas atividades, relações e ambiente do indivíduo, possibilita compreender o modelo biopsicossocial. ${ }^{22} \mathrm{~A}$ linha de cuidado baseada no modelo biopsicossocial proporciona uma atenção integral e humanizada à saúde do indivíduo, o que gera vantagens para o planejamento e ações de serviços em saúde. ${ }^{23}$

A utilização da CIF pode vir como uma alternativa ao modelo biopsicossocial pois é um dispositivo importante de gerenciamento da funcionalidade e possui potencial para nortear a ação de profissionais. ${ }^{20}$ Porém a CIF na prática clínica, com todo os seus códigos se torna inviável devido a sua extensão e ainda por necessitar de muito tempo para aplicação. A ligação da CIF com um instrumento de avaliação clínico já utilizado é uma boa proposta para sua aplicação na prática clínica, como já demonstrado em outros trabalhos, a fim de direcionar e restringir a codificação das categorias. ${ }^{8,20,24}$

A literatura demonstra que os estudos anteriores com esta metodologia conseguiram observar as potencialidades e as fraquezas de cada avaliação padronizada, levando a qualificação do cuidado. ${ }^{9,25}$

Uma limitação da presente pesquisa foi sobre a extração dos conteúdos. As fichas clínicas analisadas são compostas na sua maioria de itens "abertos" que permite uma avaliação descritiva pelo examinador. Contudo, o pesquisador tentou relacionar o conteúdo extraído da ficha com a categoria mais pertinente para ser avaliada, com o auxílio de um médico infectologista.

Por fim, a complexidade clínica das crianças expostas a zika, especialmente as com SCZ, impôs desafios para o cuidado a esta criança. As famílias e os profissionais convivem com prognósticos desconhecidos e uma avaliação clínica ampliada pode organizar a construção de plano terapêutico singular. A ficha clínica para crianças expostas ao Zika vírus analisada apresentou um olhar biomédico, com foco na doença, ao priorizar categorias de estrutura e função do corpo. Entretanto, o conhecimento já adquirido da Zika indica acometimento multissistêmicos com impacto direto no desempenho das crianças expostas, gerando uma necessidade de um olhar mais ampliado.

\section{CONCLUSÃO}

A incorporação dos domínios de atividade e participação no instrumento clínico de avaliação de um hospital especializado, beneficiará a assistência, ampliando o cuidado à saúde, de forma a elaborar perfis de funcionalidade, avaliar as necessidades e respostas a reabilitação, além de unificar a linguagem utilizada pelos profissionais de saúde o que otimiza a cooperação interprofissional e em diferentes instituições.

Refletindo dessa maneira no seguimento das crianças e suas famílias, no direcionamento de estratégias de enfrentamento, na ação das equipes de saúde envolvidas e nas políticas sociais de amparo a essa população.

\section{REFERÊNCIAS}

1. Campos GS, Bandeira AC, Sardi SI. Zika Virus Outbreak, Bahia, Brazil. Emerg Infect Dis. 2015;21(10):1885-6. Doi: https://dx.doi.org/10.3201/eid2110.150847

2. World Health Organization. Declaração da OMS sobre a primeira reunião do Comitê de Emergência sobre zika e o aumento observado em distúrbios neurológicos e malformações neonatais [texto na Internet]. Brasília (DF); OPAS Brasil; c2016 [ citado 2020 Out 20]. Disponível em: https://www.paho.org/bra/index.php?option=com cont ent\&view=article\&id=4992:declaracao-da-oms-sobre-aprimeira-reuniao-do-comite-de-emergencia-sobre-zikae-o-aumento-observado-em-disturbios-neurologicos-emalformacoes-neonatais\&Itemid=812

3. Frota L. Crianças com síndrome congênita do Zika vírus, aos 24 meses de idade: comorbidades, desenvolvimento motor grosso e percepção de mães e profissionais sobre a reabilitação [Tese]. Belo Horizonte: Universidade Federal de Minas Gerais; 2019.

4. Lopes Moreira ME, Nielsen-Saines K, Brasil P, Kerin T, Damasceno L, Pone $\mathrm{M}$, et al. Neurodevelopment in infants exposed to Zika virus in utero. $\mathrm{N}$ Engl J Med. 2018;379(24):2377-9.

https://dx.doi.org/10.1056/NEJMc1800098

5. Pone MVS, Pone SM, Zin AA, Mendes PHB, Aibe MS, Aguiar EB, et al. Zika virus infection in children: epidemiology and clinical manifestations. Childs Nerv Syst.2018;34:63-71. https://doi.org/10.1007/s00381-017-3635-3

6. Saad T, Penna e Costa AA, Góes FV, Freitas M, Almeida JV, Santa Ignêz $L$, et al. Neurological manifestations of congenital Zika virus infection. Childs Nerv Syst. 2018;34(1):73-8. Doi: https://doi.org/10.1007/s00381017-3634-4

7. Madden $\mathrm{RH}$, Bundy $\mathrm{A}$. The ICF has made a difference to functioning and disability measurement and statistics. Disabil Rehabil. 2019;41(12):1450-1462. Doi: https://doi.org/10.1080/09638288.2018.1431812 
8. Organização Mundial da Saúde. CIF: Classificação Internacional de Funcionalidade, Incapacidade e Saúde. São Paulo: EDUSP; 2020.

9. Castro SS, Castaneda L, Silveira H. Identification of common content between the questionnaire of the Health Survey (ISA-SP) and the International Classification of Functionality, Disability, and Health. Rev Bras Epidemiol. 2014;17(1):59-70. Doi: https://doi.org/10.1590/1415-790x201400010006eng

10. Piexak DR, Cezar-Vaz MR, Bonow CA. Classificação Internacional de Funcionalidade, Incapacidade e Saúde: uma Análise de conteúdo. J Res Fundam Care. 2019;11(n. esp):363-9. Doi: http://dx.doi.org/10.9789/21755361.2019.v11i2.363-369

11. Castaneda L, Bergmann A, Bahia L. A Classificação Internacional de Funcionalidade, Incapacidade e Saúde: uma revisão sistemática de estudos observacionais. Rev Bras Epidemiol. 2014;17(2):437-51. Doi: http://dx.doi.org/10.1590/1809-4503201400020012eng

12. Cieza A, Fayed N, Bickenbach J, Prodinger B. Refinements of the ICF Linking Rules to strengthen their potential for establishing comparability of health information. Disabil Rehabil. 2019;41(5):574-583.

Doi: http://dx.doi.org/10.3109/09638288.2016.1145258

13. Souza AC, Alexandre NMC, Guirardello EB. Psychometric properties in instruments evaluation of reliability and validity. Epidemiol Serv Saude. 2017;26(3):649-59. Doi: http://dx.doi.org/10.5123/S1679-49742017000300022

14. Magalhães FJ, Lima FET, Almeida PC, Ximenes LB, Chaves CMP. Protocolo de acolhimento com classificação de risco em pediatria: confiabilidade interobservadores. Acta Paul Enferm. 2017;30(3):262-70. Doi: https://doi.org/10.1590/1982-0194201700040

15. Sampaio RF, Luz MT. Funcionalidade e incapacidade humana: explorando o escopo da classificação internacional da Organização Mundial da Saúde. Cad Saude Publica. 2009;25(3):475-83. Doi: https://doi.org/10.1590/s0102-311×2009000300002

16. Cicuto Ferreira Rocha NA, Campos AC, Cicuto Ferreira Rocha F, Pereira dos Santos Silva F. Microcephaly and Zika virus: Neuroradiological aspects, clinical findings and a proposed framework for early evaluation of child development. Infant Behav Dev. 2017;49:70-82. Doi: https://doi.org/10.1016/i.infbeh.2017.07.002
17. Prata-Barbosa A, Martins MM, Guastavino AB, Cunha AJLAD. Effects of Zika infection on growth. J Pediatr (Rio J). 2019;95 Suppl 1:30-41. Doi: https://doi.org/10.1016/i.jped.2018.10.016

18. Santos DSS, Mercês MO, Souza RL, Santana AL. Cuidados à criança com síndrome congênita do Zika: revisão sistemática. REBRASF. 2018; 6(1):81-92.

19. Alves LV, Paredes CE, Silva GC, Mello JG, Alves JG. Neurodevelopment of 24 children born in Brazil with congenital Zika syndrome in 2015: a case series study. BMJ Open. 2018;8(7):e021304. Doi: https://doi.org/10.1136/bmjopen-2017-021304

20. Ferreira HNC, Schiariti V, Regalado ICR, Sousa KG, Pereira SA, Fechine CPNDS, et al. Functioning and disability profile of children with microcephaly associated with congenital Zika virus infection. Int J Environ Res Public Health. 2018;15(6):1107.

Doi:

https://doi.org/10.3390/ijerph15061107

21. Barbosa AP, Santos DT, Santos LS, Gomes RAS, Anjos CC. O uso da CIF como proposta para o acompanhamento das crianças com síndrome congênita do Zika vírus: relato de um caso 2016. Rev Cient CIF Bras. 2016;6(6):18-33.

22. Moser AD, Scharan K. O olhar biopsicossocial na Fisioterapia: ferramentas disponíveis para sua operacionalização. Fisioter Mov. 2018;31:e003136. Doi: https://doi.org/10.1590/1980-5918.031.ed01

23. Castaneda L. O Cuidado em saúde e o modelo biopsicossocial: apreender para agir. CoDAS 2019;31(5):e20180312. Doi: https://doi.org/10.1590/2317-1782/20192018312

24. Castro SS, Castaneda L, Araújo ES, Buchalla CM. Aferição de funcionalidade em inquéritos de saúde no Brasil: discussão sobre instrumentos baseados na Classificação Internacional de Funcionalidade, Incapacidade e Saúde (CIF). Rev Bras Epidemiol. 2016;19(3):679-687. Doi: https://doi.org/10.1590/1980-5497201600030018

25. Thompson SV, Cech DJ, Cahill SM, Krzak JJ. Linking the Pediatric Evaluation of Disability Inventory-Computer Adaptive Test (PEDI-CAT) to the International Classification of Function. Pediatr Phys Ther. 2018;30(2):113-118. https://doi.org/10.1097/PEP.0000000000000483 